\title{
Communication via Decentralized Processing
}

\author{
Amichai Sanderovich*,Shlomo Shamai (Shitz) ${ }^{*}$,Yossef Steinberg*and Gerhard Kramer ${ }^{\dagger}$ \\ ${ }^{*}$ Technion, Haifa, Israel \\ ${ }^{\dagger}$ Bell Laboratories, Lucent Technologies, NJ
}

\begin{abstract}
The common problem of a nomadic terminal sending information to a remote destination via agents with lossless connections is investigated. Such a setting suits, e.g. access points of a wireless network, where each access point is equipped with a different connection bandwidth. The case where these agents do not have any decoding ability is fully characterized for the Gaussian channel, when the transmitter uses "typical" codewords. For general discrete memoryless channels, lower and upper bounds are derived. An achievable rate with unrestricted agents, which are capable of decoding, is also given and then demonstrated by a numerical example for the Gaussian channel.
\end{abstract}

\section{INTRODUCTION}

The information theory of networks, and especially wireless networks, is in the focus of an extensive research activity. This interest is partly due to many recent results about the multiple antenna channel, which demonstrate significant improvements, especially for fading channels.

Many papers propose and analyze, in information theoretic terms, ad-hoc wireless networks. The relaying technique, or as it is sometimes called, multi-hopping, makes use of several intermediate wireless nodes to help the communication between two distant nodes. An information theoretic view of the relay channel was given by Cover and El Gamal in [1] for a single relay node and extended by [2] for several relaying nodes. Relaying can be coarsely divided into compress-andforward (amplify-and-forward is viewed as a special case) and decode-and-forward, depending on whether the relays decode the transmitted message or just forward the received signal to the destination. An upper bound derived by [3] suggests that as the number of users in an ad-hoc network increases, the total rate of each user tends to zero. This bound motivates the use of networks that are not solely ad-hoc, but are composed of base stations or access points as well.

The problems of conveying a source that is observed by remote agents to a single destination are built around similar settings, where the source is an i.i.d. random variable. Many problems are analyzed within an information theoretic setting, such as distributed source coding, CEO [4] and sensor networks. A connection between these rate-distortion problems to network scenarios is reported in [5],[6],[7]. The CEO setting is also addressed by tools of estimation theory (where it is commonly referred to as fusion center), such as [8],[9] and [10]. The dissertation of Schein [11] focuses on the problem of communication via two agents (parallel relays), which are connected via lossless links to the final destination, and several achievable rates are demonstrated there.
Here we consider the problem of communication through either non-decoding or decoding agents. This means that the agents use a noisy version (via their respective channels) of the transmitted message, and are able to transmit a predetermined number of bits to the destination without any errors. The destination views the source only via the agents' transmissions as in [11].

The rest of the paper is organized as follows: in section II the setting of the problem is given. An achievable rate and an upper bound are presented in sections III and IV respectively. An achievable rate for the case where the agents can decode is the focus of section V. Section VI deals with the Gaussian channel and includes a complete characterization for the case where the agents are unaware of the code used.

\section{Problem Settings}

We use capital letters for random variables, capital letters with subscripts $X_{i}$ for the $i$-th element in a random vector, and a capital letter with a superscript $X^{n}$ for the vector $\left(X_{1}, \ldots, X_{n}\right)$. The notation $X_{k}^{m}$ refers to the vector $\left(X_{k}, \ldots, X_{m}\right)$. Calligraphic letters $\mathcal{X}$ or $\mathcal{T}$ denote sets. Lowercase letters will usually be used for indices or realizations of random variables.

We consider the problem of a single transmission through $T$ agents, playing the role of decentralized processors, as is seen in Fig. 1. Our model consists of a nomadic transmitter $S$, which uses random codebooks, where the agents do not know the codebook used. Such random coding is also used in [12] for a mis-match scenario, while the advantages of random codebooks were demonstrated in [13] for unknown channels.

The following properties and definitions hold, unless stated otherwise:

1) The output of the transmitter $S$ is $X \in \mathcal{X}$ for every channel use.

2) The $T$ agents $A_{1}, \ldots, A_{T}$ receive the outputs of a memoryless broadcast channel, defined by

$$
\begin{aligned}
P_{Y_{1}^{k}, \ldots, Y_{T}^{k} \mid X^{k}}\left(Y_{1}^{k}, \ldots, Y_{T}^{k} \mid X^{k}\right)= & \\
& \prod_{i=1}^{k} P_{Y_{1}, \ldots, Y_{T} \mid X}\left(Y_{1, i}, \ldots, Y_{T, i} \mid X_{i}\right),
\end{aligned}
$$

for any $k=[1,2, \ldots]$ and where $Y_{t} \in \mathcal{Y}_{t}$. Denote $\mathcal{T} \triangleq$ $\{1, \ldots, T\}$.

3) The bandwidth $C_{t}$, in bits per channel use, characterizes the lossless link that connects the agent $A_{t}$ to the final destination $D$. 
4) The ensemble of all $N_{\mathcal{C}}=|\mathcal{X}|^{k 2^{k R}}$ codebooks with rate $R$, codeword length $k$ and input channel alphabet $\mathcal{X}$ is denoted as $\mathcal{C}$.

5) The key $F$ is an index mapping into a code from $\mathcal{C}$, so $1 \leq F \leq N_{\mathcal{C}}$.

6) The transmitter sends $X^{k}=\phi_{S, F}(M)$, where

$$
\phi_{S, F}:\left[1, \ldots, 2^{k R}\right] \rightarrow \mathcal{X}^{k},
$$

in $k$ channel uses. The function $\phi_{S, F}$ represents the coding with code $F$, and $M$ is the message to be sent, $M \in\left[1, \ldots, 2^{k R}\right]$.

7) The transmitter $S$ and the final destination $D$ randomly select the key $F$ from $N_{\mathcal{C}}$, in an initialization stage, according to the probability

$$
P_{F}(F)=\prod_{M=1}^{2^{k R}} P_{X^{k}}\left(\phi_{S, F}(M)\right),
$$

where $P_{X^{k}}\left(X^{k}\right)=\prod_{i=1}^{k} P_{X}\left(X_{i}\right)$, for some single letter probability $P_{X}(X)$. The agents are not informed about the selected key $F$.

8) The agents encode every $n \leq k$ channel outputs (where $m=k / n$ is an integer) with $T$ encoding functions:

$$
0<t \leq T, \phi_{A t}: \mathcal{Y}_{t}^{n} \rightarrow\left[1, \ldots, 2^{n C_{t}}\right]
$$

so that

$$
0<j \leq m: V_{t, j}=\phi_{A t}\left(Y_{t,(j-1) n}^{j n}\right) .
$$

9) The final destination $D$ decodes the message $M$ from the set $V_{\mathcal{T}}^{m} \triangleq\left(V_{\mathcal{T}, 1}, \ldots, V_{\mathcal{T}, m}\right)$, which consists of $T$ vectors, each of length $m=k / n$, which are sent to the destination from the $T$ agents:

$$
\hat{M}=\phi_{D, F}\left(V_{\mathcal{T}}^{m}\right)
$$

where $\phi_{D, F}:\left[1, \ldots, 2^{m \sum^{T} n C_{t}}\right] \rightarrow\left[1, \ldots, 2^{k R}\right]$.

Notice that with the knowledge of $F, X^{k}$ is uniformly distributed over $2^{k R}$ codewords, while without the key, $X^{k}$ is distributed according to $\prod_{i=1}^{k} P_{X}\left(X_{i}\right)$. We use the following simple lemma in the sequel:

Lemma 1: Without the key $F$, the received vector $X^{k}$ is distributed according to $P_{X^{k}}\left(X^{k}\right)=\prod_{i=1}^{k} P_{X}\left(X_{i}\right)$, and therefore $Y_{t}^{k}$ are also distributed as

$$
P_{Y_{t}^{k}}\left(Y_{t}^{k}\right)=\prod_{i=1}^{k} \sum_{X} P_{Y_{t} \mid X}\left(Y_{t, i} \mid X\right) P_{X}(X) .
$$

This lemma is proved in [14].

It is easy to see from the probability laws and the fact that all possible codebooks are considered, that for a given $F$, the code is a random code chosen according to the probability law $P_{X^{k}}\left(X^{k}\right)=\prod_{i=1}^{k} P_{X}\left(X_{i}\right)$.

The above setting models the problem where the final destination decodes the message from the transmitter via simple agents, which are not able to decode the transmitted message and use compression of the received signals.

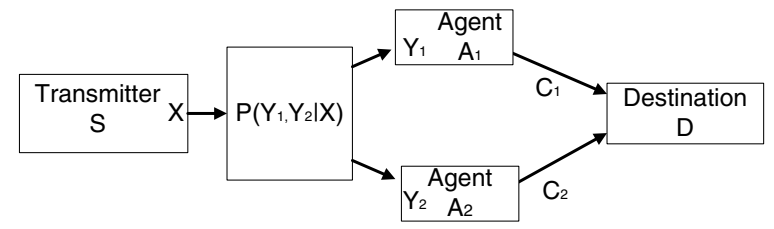

Fig. 1. A system with two agents between the transmitter and the destination.

\section{AN ACHIEVABLE RATE}

We denote the model which is described by the setting of section II as nomadic transmitter. The following theorem is a special case of theorem 3 (proved in [14], see also [2]).

Theorem 1: Define a positive $R$ such that (8) is fulfilled

$$
R<\max I\left(X ; U_{\mathcal{T}}\right)
$$

with the constraints (9) on $U_{\mathcal{T}}$

$$
\forall \mathcal{S} \subseteq \mathcal{T}: \sum_{t \in \mathcal{S}} C_{t}>I\left(U_{\mathcal{S}} ; Y_{\mathcal{S}} \mid U_{\mathcal{S}^{C}}\right)
$$

and the joint distribution (10)

$$
\begin{aligned}
& P_{X, U_{\mathcal{T}}, Y_{\mathcal{T}}}\left(X, U_{\mathcal{T}}, Y_{\mathcal{T}}\right)= \\
& P_{X}(X) P_{Y_{\mathcal{T}} \mid X}\left(Y_{\mathcal{T}} \mid X\right) \prod_{t=1}^{T} P_{U_{t} \mid Y_{t}}\left(U_{t} \mid Y_{t}\right) .
\end{aligned}
$$

Then a rate $R$ is achievable for the case of nomadic transmitter.

The probability space (10) results with the Markov relations:

$$
U_{t}-Y_{t}-\left\{X, U_{\mathcal{T} \backslash t}, Y_{\mathcal{T} \backslash t}\right\} .
$$

Since this achievable rate is attained in theorem 3 through the use of compression which is independent of the message index and the codebook used by the transmitter, theorem 1 is indeed a special case of theorem 3 .

\section{AN OUTER BOUND}

We start by stating the maximum rate $R$ so error free decoding is possible at the destination, when the agents' encoding functions are given. Using Fano's inequality, an error free decoding at the destination is possible only if:

$$
H\left(M \mid V_{\mathcal{T}}^{m}, F\right) \leq k \epsilon_{k},
$$

where $k \epsilon_{k} \rightarrow 0$ as $k \rightarrow \infty$.

Defining $\mathbf{X}_{j} \triangleq\left(X_{(j-1) n+1}, \ldots, X_{j n}\right)$, we now have:

$$
\begin{aligned}
k R & =H(M)=I\left(V_{\mathcal{T}}^{m}, F ; M\right)+H\left(M \mid V_{\mathcal{T}}^{m}, F\right) \\
& \leq I\left(V_{\mathcal{T}}^{m} ; M \mid F\right)+k \epsilon_{k} \\
& =I\left(V_{\mathcal{T}}^{m} ; \mathbf{X}^{k}(M, F) \mid F\right)+k \epsilon_{k} \\
& \leq \sum_{j=1}^{m}\left[H\left(V_{\mathcal{T}, j} \mid F\right)-H\left(V_{\mathcal{T}, j} \mid \mathbf{X}_{j}, F\right)+n \epsilon_{k}\right] \\
& \leq m \max _{P_{X^{n}\left(X^{n}\right)} I\left(V_{\mathcal{T}} ; X^{n} \mid F\right)+k \epsilon_{k},}
\end{aligned}
$$


where (14) follows since $F$ is independent of $M$ so $H(F \mid M)=H(F)$, and (16) is due to properties II - 2 and II - 8. From (17) we conclude that the transmission rate $R$ is upper bounded by

$$
R \leq \max _{P_{X^{n}}\left(X^{n}\right)} \frac{1}{n} I\left(V_{\mathcal{T}} ; X^{n} \mid F\right) .
$$

Next we would like to upper bound

$$
\frac{1}{n} I\left(V_{\mathcal{T}} ; X^{n} \mid F\right)
$$

By defining $U_{t, i} \triangleq\left(V_{t}, Y_{\mathcal{T}}^{i-1}, X^{i-1}\right)$, we get to the following theorem, which is proved in [14], using techniques similar to the ones used to show theorem 2 of [15]:

Theorem 2: The maximum reliable communication rate for a nomadic transmitter is upper bounded by

$$
R \leq \max I\left(X ; U_{\mathcal{T}}\right) .
$$

Here $U_{\mathcal{T}}$ must satisfy the constraints:

$$
\forall \mathcal{S} \subseteq \mathcal{T}: \sum_{t \in \mathcal{S}} C_{t} \geq I\left(U_{\mathcal{S}} ; Y_{\mathcal{T}} \mid U_{\mathcal{S}^{C}}\right)
$$

and also fulfill the Markov relations:

$$
U_{t}-Y_{t}-\left\{X, Y_{\mathcal{T}} \backslash t\right\}
$$

\section{Agents With CODE KNOWLEDGE}

In this section we diverge from the nomadic model described in section II. Suppose the agents know the codebook so that the agents and the transmitter can be jointly optimized. This enables to transmit a broadcast message that is decoded by the agents and forwarded to the destination, in addition to the compression operation. Denote this model as decoding agents. Such generalized approach might increase the overall transmission rate.

In the following, we will denote all messages that are decoded at the agents as broadcast messages, although they are always intended for the same final destination.

Denote by $M_{t}$ the message to be decoded at agent $t$, so that now $M=\left(M_{\mathcal{T}}, M_{C F}\right)\left(M_{C F}\right.$ is the message that is decoded only at the final destination). Since the agents have decoding ability, we assume that they also process much longer output sequences, so that now $n=k$, and we can drop the $k$ index altogether.

Theorem 3: For the decoding agents case, any rate $R$ satisfying (23)

$$
R<I\left(X ; U_{\mathcal{T}} \mid W_{\mathcal{T}}\right)+\sum_{t=1}^{T} R_{B C, t},
$$

with the constraints (24) on $R_{B C, \mathcal{T}}, U_{\mathcal{T}}, W_{\mathcal{T}}$ (W $W_{0}$ is a constant)

$$
\left\{\begin{array}{l}
\forall 0<t \leq T: 0 \leq R_{B C, t}<\min \left\{I\left(W_{t} ; Y_{t}\right), C_{t}\right\} \\
\forall \mathcal{S} \subseteq \mathcal{T}: \\
\sum_{t \in \mathcal{S}}\left[C_{t}-R_{B C, t}\right]>I\left(U_{\mathcal{S}} ; Y_{\mathcal{S}} \mid U_{\mathcal{S} C}, W_{\mathcal{T}}\right) \\
\sum_{t \in \mathcal{S}} R_{B C, t}<\sum_{t \in \mathcal{S}}\left[I\left(W_{t} ; Y_{t}\right)-I\left(W_{t} ; W_{\tilde{\mathcal{T}}(\mathcal{S}, t)}\right)\right]
\end{array}\right.
$$

when $\tilde{\mathcal{T}}(\mathcal{S}, t) \triangleq\{i: \quad i \in \mathcal{S}$ and $i<t\}$ and the joint distribution (25)

$$
\begin{aligned}
P_{X, Y_{\mathcal{T}}, W_{\mathcal{T}}, U_{\mathcal{T}}}\left(X, Y_{\mathcal{T}}, W_{\mathcal{T}}, U_{\mathcal{T}}\right)= \\
P_{W_{\mathcal{T}}}\left(W_{\mathcal{T}}\right) P_{X \mid W_{\mathcal{T}}}\left(X \mid W_{\mathcal{T}}\right) P_{Y_{\mathcal{T}} \mid X}\left(Y_{\mathcal{T}} \mid X\right) \\
\cdot \prod_{t=1}^{T} P_{U_{t} \mid Y_{t}, W_{t}}\left(U_{t} \mid Y_{t}, W_{t}\right),
\end{aligned}
$$

is achievable.

The agent $A_{t}$ forwards $n R_{B C, t}$ decoded bits to the destination along with $n\left(C_{t}-R_{B C, t}\right)$ bits for the compression. The achievable rate may be further increased to the convex hull of (23) and (24).

Proof: The proof appears in [14] and uses compression in addition to Marton's scheme (not including common messages).

Remark 1: The above achievable rate can be increased by sending only partial messages to the final destination. This means in fact doing Slepian Wolf (SW) distributed compression of the messages to the final destination.

Remark 2: Another improvement upon (23) is done by sending common broadcast messages in addition to the individual broadcast messages to the agents. This is achieved by extending theorem 2 in [16] to more than two users and including compression. Notice that such construction contains theorem 3 and theorem 4 (to follow) as special cases.

Remark 3: The scheme described in theorem 1 is obtained as a special case of the above scheme, by taking all $W_{\mathcal{T}}$ to be constants.

For the case where the channels $P_{Y_{t} \mid X}\left(Y_{t} \mid X\right)$ are either stochastically or physically degraded [17], that is $Y_{t}$ is better than $Y_{t-1}$ (without loss of generality), we can use superposition coding, which is known to achieve capacity for degraded broadcast channels. The received signal $Y_{2}$ is physically degraded compared to $Y_{1}$ if the following Markov chain

$$
X-Y_{1}-Y_{2}
$$

is satisfied. Notice that this relation leaves $I\left(X ; Y_{1}, Y_{2}\right)=$ $I\left(X ; Y_{1}\right)$. On the other hand, stochastically degraded $Y_{2}$ means that the marginal probability $P_{Y_{2} \mid X}\left(Y_{2} \mid X\right)$ can be calculated from $P_{Y_{1} \mid X}\left(Y_{1} \mid X\right)$ through some $P_{Y_{2} \mid Y_{1}}\left(Y_{2} \mid Y_{1}\right)$. Notice that since (26) is not necessarily true for stochastic degradedness, we have that $I\left(X ; Y_{1}, Y_{2}\right) \geq I\left(X ; Y_{1}\right)$. So although superposition coding is optimal for the degraded broadcast channel, it is not optimal, in general, for our model.

Theorem 4: For decoding agents with a channel $P_{Y_{\mathcal{T}} \mid X}\left(Y_{\mathcal{T}} \mid X\right)$ that satisfies stochastic degradedness (27),

$$
\begin{aligned}
\forall 0<t \leq T: \quad & P_{Y_{t-1} \mid X}\left(Y_{t-1} \mid X\right)= \\
& \sum_{Y_{t}} P_{Y_{t} \mid X}\left(Y_{t} \mid X\right) P_{Y_{t-1} \mid Y_{t}}\left(Y_{t-1} \mid Y_{t}\right),
\end{aligned}
$$

any rate $R$ satisfying (23) with the constraints (28) on 


$$
\begin{aligned}
& R_{B C, \mathcal{T}}, U_{\mathcal{T}}, W_{\mathcal{T}}\left(W_{0} \text { is a constant }\right) \\
& \left\{\begin{array}{l}
\forall 0<t \leq T: 0 \leq R_{B C, t} \leq C_{t} \\
\forall 0<t \leq T: \sum_{i=1}^{t} R_{B C, i}<\sum_{i=1}^{t} I\left(W_{i} ; Y_{i} \mid W^{i-1}\right) \\
\mathcal{S} \subseteq \mathcal{T}: \sum_{t \in \mathcal{S}} C_{t}-R_{B C, t}>I\left(U_{\mathcal{S}} ; Y_{\mathcal{S}} \mid U_{\mathcal{S}^{C}}, W_{\mathcal{T}}\right),
\end{array}\right.
\end{aligned}
$$

and the joint distribution (29)

$$
\begin{array}{r}
P_{X \mid W_{\mathcal{T}}}\left(X \mid W_{\mathcal{T}}\right) P_{Y_{\mathcal{T}} \mid X}\left(Y_{\mathcal{T}} \mid X\right) \prod_{t=1}^{T} P_{U_{t} \mid Y_{t}, W^{t}}\left(U_{t} \mid Y_{t}, W^{t}\right) \\
\cdot \prod_{t=1}^{T} P_{W_{t} \mid W^{t-1}}\left(W_{t} \mid W^{t-1}\right),
\end{array}
$$

is achievable.

Theorem 3 does not seem to include theorem 4 as a special case, as it does not account for the common rate in Marton's region.

\section{THE GAUSSIAN CHANNEL}

In this section we investigate the Gaussian channel under two cases, where the agents are ignorant about the code and where they are cognizant of the codebook used by the transmitter. Using the latest results of Tse et. al., for the Gaussian CEO rate-distortion problem [18], a converse for the reliable communication rate is shown for the former case. We use the results of theorem 1 with continuous alphabets, where the extension relies on standard arguments.

\section{A. Non-decoding agents}

The Gaussian channel is defined by $Y_{t}=X+N_{t}$, where $N_{t}$ are independent Gaussian random variables with $\mathrm{E} N_{t}^{2}=$ $P_{N_{t}}{ }^{1}$ and $\mathrm{E} N_{t}=0$. Let $P_{X}(X)$ be zero mean Gaussian with variance $\mathrm{E} X^{2}=P_{X}$.

We prove the following result.

Theorem 5: The capacity of the nomadic transmitter, for the Gaussian channel (described above), is

$$
\begin{aligned}
& R=\max _{r_{t} \geq 0} \min _{\mathcal{S} \subseteq \mathcal{T}} \\
& \quad\left\{\frac{1}{2} \log _{2}\left(1+P_{X} \sum_{t \in \mathcal{S}^{C}} \frac{1-2^{-2 r_{t}}}{P_{N_{t}}}\right)+\sum_{t \in \mathcal{S}}\left[C_{t}-r_{t}\right]\right\} .
\end{aligned}
$$

The theorem is proved in [14] using the technique of [18]. In the sequel, the constants $r_{t}$ are interpreted as rates associated with noise quantization [18]. The capacity is determined by calculating the sufficient bandwidths $\underline{C} \triangleq\left(C_{1}, \ldots, C_{T}\right)$ for communication at rate $R$, and by showing that it equals the necessary bandwidths $\bar{C} \triangleq\left(C_{1}, \ldots, C_{T}\right)$ for reliable communication with rate $R$.

Proof: The altered proof is briefly sketched here, for completeness.

First, the sum-bandwidths under the constraints

$$
\sum_{t=1}^{m} C_{t} \leq a_{m}, m=1, \ldots, T-1
$$

\footnotetext{
${ }^{1} \mathrm{E}$ stands for statistical expectation
}

is shown to be ( $\left.\bar{a} \triangleq\left\{a_{1}, \ldots, a_{T-1}\right\}\right)$

$$
C_{\min }\left(a_{1}, \ldots, a_{T-1}\right)=R+\min _{r_{\mathcal{T}} \in \nabla(R, \bar{a})} \sum_{t=1}^{T} r_{t},
$$

where the space $\nabla(R, \bar{a})$ is defined as $r_{\mathcal{T}}$ that satisfy the following $2 T$ conditions:

$$
\left\{\begin{array}{l}
\forall 0<t \leq T: 0 \leq r_{t} \\
\forall 0<m \leq T-1: \\
a_{m} \geq R-\frac{1}{2} \log _{2}\left(1+P_{X} \sum_{t=m+1}^{T} \frac{1-2^{-2 r_{t}}}{P_{N_{t}}}\right)+\sum_{t=1}^{m} r_{t} \\
R=\frac{1}{2} \log _{2}\left(1+P_{X} \sum_{t=1}^{T} \frac{1-2^{-2 r_{t}}}{P_{N_{t}}}\right) .
\end{array}\right.
$$

Next, the above sum-bandwidth identity leads to identical bandwidth-regions $(\underline{C}(R) \leq \bar{C}(R))$. This is since both regions are convex [18] and since for all non-negative vectors $\left(\alpha_{1}, \ldots, \alpha_{T}\right)$ :

$$
\min _{\left(C_{1}, \ldots, C_{T}\right) \in \bar{C}(R)} \sum_{t=1}^{T} \alpha_{t} C_{t} \geq \min _{\left(C_{1}, \ldots, C_{T}\right) \in \underline{C}(R)} \sum_{t=1}^{T} \alpha_{t} C_{t},
$$

where (34) is proved in [18].

\section{B. Example: agents with decoding capabilities}

Consider the symmetric case of a Gaussian channel with statistically equivalent agents (both suffering from an additive Gaussian noise with variance $P_{N}$ ). In addition, both agents are connected via lossless links with equal bandwidth $C$, to the final destination. The combined approach of broadcast and compression for the degraded channel (theorem 4) is employed. The rate $R$ is achievable provided that:

$$
R<\sum_{t=1}^{2} R_{t}+\frac{1}{2} \log _{2}\left(1+\alpha \frac{P_{X}}{P_{N}} \sum_{t=1}^{2}\left(1-e^{-2 r_{t}}\right)\right)
$$

where the $\left\{r_{t}, R_{t}, \alpha\right\}$ satisfy:

$$
\left\{\begin{array}{l}
0 \leq \alpha \leq 1 \\
t=1,2: \quad 0 \leq R_{t} \leq C \\
\sum_{t=1}^{2} R_{t}<\frac{1}{2} \log _{2}\left(\frac{P_{N}+P_{X}}{P_{N}+\alpha P_{X}}\right) \\
\forall \mathcal{S} \subseteq\{1,2\}: \\
\sum_{\mathcal{S}}\left[C-R_{t}\right]>\sum_{\mathcal{S}} r_{t}+ \\
\quad \frac{1}{2} \log _{2}\left(1+\alpha P_{X} \sum_{t=1}^{2} \frac{1-2^{-2 r_{t}}}{P_{n}}\right)+ \\
\quad-\frac{1}{2} \log _{2}\left(1+\alpha \frac{P_{X}}{P_{n}} \sum_{S^{C}}\left(1-2^{-2 r_{t}}\right)\right) .
\end{array}\right.
$$

The convex hull is found to improve rates for this example. The achievable rate as a function of the bandwidth $C$, for signal to noise ratio $\frac{P_{X}}{P_{n}}=10$, is presented in Fig. 2. In this figure, the left most dashed line $R=2 C$, and the upper flat dashed line $R=\frac{1}{2} \log _{2}\left(1+2 \frac{P_{X}}{P_{n}}\right)$ are the two cut-set bounds [17], and the lower flat dashed line is the rate of a system without compression $R=\frac{1}{2} \log _{2}\left(1+\frac{P_{X}}{P_{n}}\right)$. The dotted line represents time-sharing, which is useful here. This figure illustrates that if the sum of capacities of the corresponding broadcast channel, (calculated by the signal to noise ratios 


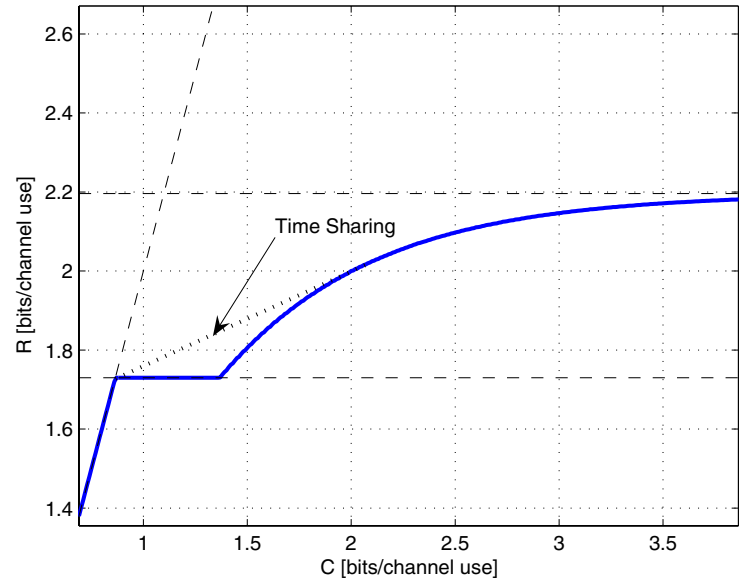

Fig. 2. The achievable rate of a system with two agents, each with link bandwidth of $C$ and signal to noise ratio of $10 \mathrm{~dB}$. The dotted line designates time sharing, and the dashed lines represent the cut-set bounds [17]. The lower flat dashed line is the achievable rate for a system without compression.

at the agents) is smaller than the sum of the bandwidths of the links, a compression scheme can significantly improve the performance. A rate of up to 0.2 bits from the cut-set bound is observed with $C=R=2$, which means $50 \%$ excess bandwidth compared to the achievable rate.

\section{CONCLUSION}

Communication via separated agents is considered, focusing on two cases: (1) the agents do not possess any knowledge about the codebook used by the transmitter, and (2) the agents do possess decoding capability. For the first case, a direct coding theorem based on decentralized quantization and an outer bound were presented. Considering the Gaussian channel, a converse was proved by the entropy power inequality invoking the results of [18]. An achievable rate was derived also for the case where the agents are cognizant of the codebook used by the transmitter. These achievability arguments combine Marton's achievable rates for the broadcast channel with decentralized quantization. The utility of time sharing was demonstrated in the Gaussian example.

\section{ACKNOWLEDGMENT}

This research was supported by the EU 6th framework program via the NEWCOM network of excellence. The authors are indebt to Mr. Michael Peleg for helpful discussions.

\section{REFERENCES}

[1] T. M. Cover and A. A. El-Gamal, "Capacity theorems for the relay channel," IEEE Trans. Inform. Theory, vol. 25, no. 5, pp. 572-584, Jan 1979.

[2] G. Kramer, M. Gastpar, and P. Gupta, "Information-theoretic multihopping for relay networks," in International Zürich seminar on communication, Swizerland, Feb. 2004.

[3] O. Lévêque and E. Telatar, "Information theoretic upper bounds on the capacity of large extended ad hoc wireless networks," IEEE Trans. Inform. Theory, vol. 51, no. 3, pp. 858-865, Mar. 2005.

[4] T. Berger, Z. Zhang, and H. Viswanathan, "The CEO problem," IEEE Trans. Inform. Theory, vol. 42, no. 3, pp. 887-902, May 1996

[5] Y. Steinberg and N. Merhav, "On successive refinement for the WynerZiv problem," IEEE Trans. Inform. Theory, vol. 50, no. 8, pp. 16361654, Aug. 2004.

[6] J. Barros and S. D. Servetto, "A note on cooperative multiterminal source coding," in the Proceedings of the 38th Annual Conference on Information Sciences and Systems (CISS), Princeton, NJ, Mar. 2004.

[7] A. D. Murugan, P. K. Gopala, and H. El-Gamal, "Correlated sources over wireless channels: cooperative source-channel coding," IEEE J. Select. Areas Commun., vol. 22, no. 6, pp. 988-999, Aug. 2004.

[8] Z.-Q. Luo, "Universal decentralized estimation in a bandwidth constrained sensor network," IEEE Trans. Inform. Theory, vol. 51, no. 6, pp. 2210-2219, June 2005 .

[9] J. A. Gubner, "Distributed estimation and quantization," IEEE Trans. Inform. Theory, vol. 39, no. 4, pp. 1456-1459, July 1993.

[10] M. Longo, T. D. Lookabaugh, and R. M. Gray, "Quantization for decentralized hypothesis testing under communication constraints," IEEE Trans. Inform. Theory, vol. 36, no. 2, pp. 241-255, Mar. 1990.

[11] B. E. Schein, "Distributed coordination in network information theory," Ph.D. dissertation, MIT, October 2001

[12] S. Shamai and A. Lapidoth, "Fading channels: How perfect need "perfect side information" be?" IEEE Trans. Inform. Theory, vol. 48, no. 5, pp. 1118-1134, May 2002.

[13] I. G. Stiglitz, "Coding for a class of unknown channels," IEEE Trans. Inform. Theory, vol. IT-12, no. 2, pp. 189-195, April 1966.

[14] A. Sanderovich, S. Shamai, Y. Steinberg, and G. Kramer, "Communication via decentralized processing," in preparation.

[15] J. Chen, X. Zhang, T. Berger, and S. B. Wicker, "An upper bound on the sum rate distortion function and its corresponding rate allocation schemes for the CEO problem," IEEE J. Select. Areas Commun., vol. 22 no. 6, pp. 977-987, Aug. 2004.

[16] K. Marton, "A coding theorem for the discrete memoryless broadcast channel," IEEE Trans. Inform. Theory, vol. IT-25, pp. 306-311, May 1979.

[17] T. M. Cover and J. A. Thomas, Elements of Information theory. John Wiley \& Sons, Inc., 1991.

[18] V. Prabhakaran, D. Tse, and K. Ramchandran, "Rate region of the quadratic Gaussian CEO problem," in Proc IEEE Int Symp Info Theory ISIT2004, Chicago, IL, June 2004, p. 119. 